\title{
On solving Schwinger-Dyson equations for non-Abelian gauge theory
}

\author{
V.E. Rochev* \\ Institute for High Energy Physics \\ 142284 Protvino, Moscow region, Russia
}

July 29, 2018

\begin{abstract}
A method for solving Schwinger-Dyson equations for the Green function generating functional of non-Abelian gauge theory is proposed. The method is based on an approximation of Schwinger-Dyson equations by exactly soluble equations. For the $S U(2)$ model the first step equations of the iteration scheme are solved which define a gauge field propagator. Apart from the usual perturbative solution, a nonperturbative solution is found, which corresponds to the spontaneous symmetry breaking and obeys infrared finite behaviour of the propagator.
\end{abstract}

\section{Introduction}

The Schwinger-Dyson equations (SDE) method is one of the basic tools for investigating of the Green functions of the quantum theory. Hitherto the one universal method for solving SDEs has been the coupling constant perturbation theory (hereafter simply termed perturbation theory). The range of applicability of other methods (for example, $1 / N$-expansion) is limited by a narrow class of models. In

\footnotetext{
${ }^{0}$ rochev@mx.ihep.su
} 
particular, the $1 / N$-expansion method cannot be used in the calculations for non-Abelian gauge theories due to the complicated structure of the leading approximation.

On the other hand, the applicability of perturbation theory to the investigation of non-Abelian gauge theories is limited by a deepEuclidean region. In the non-perturbative region of small momenta the physical vacuum of non-Abelian gauge theories obeys the nontrivial structure that is beyond the framework of perturbation theory. In the SDE terms this fact can be understood if one takes into account the radical difference in the properties of the leading-approximation equations of the perturbation theory and the original exact equations. The SDEs for the generating functional of Green functions are equations in functional derivatives. The leading approximation of perturbation theory comprises neglecting the higher derivatives terms in these equations (just such terms correspond to an interaction). The leading-approximation equations of perturbation theory are of a lower order compared with the exact ones; therefore, the class of solutions thus described contracts drastically, and non-perturbative solutions which correspond to the nontrivial physical vacuum practically drop out of the consideration. This feature of SDEs in the non-perturbative region is noted repeatedly in simple models (see [1]).

In this work a method for the SDE solution of non-Abelian gauge theory is proposed. It takes into account the terms with higher derivatives (i.e. self-interaction of the non-Abelian fields) $a b$ ovo in the leading approximation. Though we limit ourselves to the simplest extension of the class of SDE solutions, the results are non-trivial: the non-perturbative solution which corresponds to spontaneous symmetry breaking (with non-Higgs mechanism) is found. It obeys a nonsingular behaviour of the propagator in the non-perturbative infrared region of small momenta.

An idea of the method comprises the approximation of SDEs for the generating functional by equations with "constant" (i.e. independent of sources) coefficients. These equations have a simple exponential solution, which is a foundation for the linear iteration scheme. The method is universal, as is perturbation theory, i.e. it is applicable to practically any model of quantum field theory. For the scalar $\phi^{4}$ theory it has been shown in [2] that the method describes such non-perturbative phenomena as spontaneous symmetry breaking and the trivialization of $\phi^{4}$ theory at $d=4$. This method has also been 
successfully applied in the investigation of the Gross-Neveu model for finite $N$ [3].

\section{Schwinger-Dyson equations and the iteration scheme}

A system of SDEs for the generating functional $G(J, \eta)$ of the Green functions of non-Abelian gauge theory has the form

$$
\begin{gathered}
\mathcal{D}_{\nu}^{a b}\left(\frac{\delta}{i \delta J}\right) F_{\nu \mu}^{b}\left(\frac{\delta}{i \delta J}\right) G+\frac{1}{\alpha} \partial_{\mu} \partial_{\nu} \frac{\delta G}{i \delta J_{\nu}^{a}}+g f^{a b c} \frac{\delta}{\delta \bar{\eta}^{c}} \partial_{\mu} \frac{\delta G}{\delta \eta^{b}}+J_{\mu}^{a} G=0, \\
i \partial_{\mu} \mathcal{D}_{\mu}^{a b}\left(\frac{\delta}{i \delta J}\right) \frac{\delta G}{\delta \bar{\eta}^{b}}+\eta^{a} G=0 .
\end{gathered}
$$

Here $F_{\mu \nu}^{a}(A)=\partial_{\mu} A_{\nu}^{a}-\partial_{\nu} A_{\mu}^{a}+g f^{a b c} A_{\mu}^{b} A_{\nu}^{c}$ is the gauge field tensor, $\mathcal{D}_{\mu}^{a b}(A)=\delta^{a b} \partial_{\mu}-g f^{a b c} A_{\mu}^{c}$ is the covariant derivative, $f^{a b c}$ are structure constants of a gauge group, $J_{\mu}^{a}(x)$ is the source of the gauge field, $\eta^{a}(x)$ is the source of a ghost field, $\alpha$ is the gauge parameter, $g$ is the coupling constant. We work in Minkowski space with a metric $(1,-1,-1,-1)$, and $x_{\mu} y_{\mu} \equiv g_{\mu \nu} x^{\mu} y^{\nu}$ by definition.

At $g=0$ the system (1)-(2) has a solution

$$
G_{\text {free }}=\exp \left\{\frac{1}{2 i} J * D_{\text {free }} * J+i \bar{\eta} * \Delta_{\text {free }} * \eta\right\},
$$

where $D_{\text {free }}$ and $\Delta_{\text {free }}$ are free propagators of the gauge field and ghost field respectively, and

$$
J * D_{\text {free }} * J \equiv \int d x d y J_{\mu}^{a}(x)\left(D_{\text {free }}\right)_{\mu \nu}^{a b}(x-y) J_{\nu}^{a}(y),
$$

etc. The functional $G_{\text {free }}$ is the generating functional of the free Green functions. The iteration scheme based on $G_{\text {free }}$ is the usual coupling constant perturbation theory.

We shall use an alternative iteration scheme for SDEs (1)-(2), which is formulated as follows: the leading approximation is a system of equations with terms proportional to the sources $J$ and $\eta$ omitted. (For the system of eqs. (1) and (2) these are the last terms.) This system has a solution

$$
G_{0}=\exp i\{J * V+\bar{\eta} * C+\bar{C} * \eta\},
$$


where $J * V \equiv \int d x J_{\mu}^{a}(x) V_{\mu}^{a}(x)$, etc. The coefficient functions $V_{\mu}^{a}$ and $C^{a}$ are solutions of the corresponding system of "characteristic equations". When constructing the iteration scheme for the generating functional

$$
G=G_{0}+G_{1}+\cdots+G_{n}+\cdots
$$

the omitted terms $J G$ and $\eta G$ should be considered as perturbations, i.e. equations of the iteration scheme are

$$
\begin{gathered}
\left\{\mathcal{D}_{\nu}\left(\frac{\delta}{i \delta J}\right) F_{\nu \mu}\left(\frac{\delta}{i \delta J}\right)+\frac{1}{\alpha} \partial_{\mu} \partial_{\nu} \frac{\delta}{i \delta J_{\nu}}+g f \frac{\delta}{\delta \bar{\eta}} \partial_{\mu} \frac{\delta}{\delta \eta}\right\} G_{n}=-J_{\mu} G_{n-1} \\
i \partial_{\mu} \mathcal{D}_{\mu}\left(\frac{\delta}{i \delta J}\right) \frac{\delta G_{n}}{\delta \bar{\eta}}=-\eta G_{n-1}
\end{gathered}
$$

A solution of eqs.(3)-(4) has the form $G_{n}=P_{n} G_{0}$, where $P_{n}$ is a polynomial in $J$ and $\eta$. Therefore, at each iteration step we obtain a closed system of equations for coefficient functions of the polynomial $P_{n}$, which completely defines the Green functions of the given step. There is no manifest small parameter in the usual sense in this scheme: "smallness" is defined by the condition that the Green functions are derivatives of the generating functional at $J=\eta=0$, and it is sufficient for us to know $G(J, \eta)$ near zero, i.e. in the region where the neglected terms are small. At each step of the iteration we approximate the functional $G / G_{0}$ by a sum of the polynomials $P_{n}$, and a degree of the polynomial increases with each step. As is known, for ordinary differential equations the scheme of this type is equivalent to iterations of Volterra-type integral equations and gives an expansion that converge well. That is why we may hope that this scheme possesses good convergence properties. In any case it is clear that the convergence of this scheme is no worse than that of the perturbation theory. The perturbation theory is singular in the sense of differential equation theory, since the higher derivatives are omitted in the leading approximation. In contrast to the singular perturbation theory, the scheme proposed is regular in the sense above. This circumstance gives us hope for improving the convergence properties (see [4] for more discussion).

To remove the ultraviolet divergences it is necessary to supplement SDEs (1)-(2) and the iteration scheme equations (3)-(4) with the corresponding counterterms. The counterterms are also defined by the iteration procedure: $\delta z=\delta z_{0}+\delta z_{1}+\cdots$, i.e. at each step it is 
necessary to take into account the counterterms of the corresponding order.

Let us consider the leading approximation in more detail. As has been noted above the solution of the leading approximation equations is the linear exponential in the sources. The vacuum structure is defined by the solutions of the characteristic equations for $V_{\mu}^{a}$ and $C^{a}$

$$
\begin{aligned}
\mathcal{D}_{\nu}(V) F_{\nu \mu}(V)+\frac{1}{\alpha} \partial_{\mu} \partial_{\nu} V_{\nu}+g f C \partial_{\mu} \bar{C} & =0, \\
\partial_{\mu} \mathcal{D}_{\mu}(V) C & =0 .
\end{aligned}
$$

These equations have a great number of solutions which reflects the non-trivial vacuum structure of the non-Abelian gauge theory. In this connection it is appropriate to recall 't Hooft's conjecture [5] on the existence of different vacuum modes for non-Abelian gauge theory at zero temperature: a superconducting one (spontaneously broken), dual superconducting (confinement), etc. Realization of either mode depends on the values of some quantities (manager parameters) whose definition is a problem of the quantum field dynamics. It is reasonable to suppose that the whole set of solutions of characteristic equations (5) is divided into classes, each corresponds to some mode of the gauge theory in the above sense .

In this paper we limit ourselves to investigation of the first step of the iteration scheme based on the simplest solutions of the characteristic equations (5).

The trivial solution of eqs.(5) $V_{\mu}^{a}=C^{a}=0$ (i.e., $G_{0}=1$ ) leads to an iteration scheme which is simply the reconstructed perturbation theory. The simplest extension of the set of vacuum solutions compared with the trivial perturbative vacuum comprises taking into account non-zero constant solutions of eqs.(5). Namely, we choose the leading approximation in the form

$$
G_{0}=\exp i\{J * V\},
$$

where the vacuum vectors $V_{\mu}^{a}$ are independent of the space-time variables and satisfy the condition

$$
f^{a b c} f^{c d h} V_{\nu}^{b} V_{\nu}^{d} V_{\mu}^{h}=0
$$

which follows from the characteristic equations (5), if $C=\partial V=0$. Below we shall consider only this class of the leading approximation 
solutions. Surely we have no prior physical foundation (except for reasons of simplicity) for the choice of this class of solutions. Nevertheless, we shall see that this simple set of solutions leads to a new spontaneously broken mode.

Concluding our discussion of the leading approximation, let us touch upon the definition of the ground state ("physical vacuum"). Due to the linearity of SDEs (1)-(2) and the iteration scheme equations (3)-(4) an arbitrary linear combination of solutions is also a solution. Therefore, the physical vacuum functional $G=<0 \mid 0>_{J}$ should be constructed as a linear combination of partial solutions, each of them corresponding to some solution of the characteristic equations (5). In other words, the physical vacuum is a superposition of $V$-vacua. This combination should be chosen in such a way as to maintain the admissible physical properties of the Green functions (for example, $<0\left|A_{\mu}^{a}\right| 0>=0$ ) and the energy minimality condition.

To formulate the iteration scheme in terms of the polynomials $P_{n}$, it is convenient to introduce the matrix quantity

$$
W_{\mu}^{a b}=i g f^{a b c} V_{\mu}^{c} .
$$

Then, the iteration scheme equations have the form of equations for the polynomials $P_{n}$ :

$$
\begin{gathered}
\left\{\left[\mathcal{D}_{\nu}(V)+i g f \frac{\delta}{\delta J_{\nu}}\right]\left[F_{\nu \mu}\left(\frac{\delta}{i \delta J}\right)+W_{\nu} \frac{\delta}{\delta J_{\mu}}-W_{\mu} \frac{\delta}{\delta J_{\nu}}\right]+\right. \\
\left.i\left[W_{\mu}, W_{\nu}\right] \frac{\delta}{\delta J_{\nu}}+\frac{1}{\alpha} \partial_{\mu} \partial_{\nu} \frac{\delta}{i \delta J_{\nu}}+g f \frac{\delta}{\delta \bar{\eta}} \partial_{\mu} \frac{\delta}{\delta \eta}\right\} P_{n}=-J_{\mu} P_{n-1} \\
\partial_{\mu}\left[\mathcal{D}_{\mu}(V)+i g f \frac{\delta}{\delta J_{\mu}}\right] \frac{\delta P_{n}}{\delta \bar{\eta}}=i \eta P_{n-1} .
\end{gathered}
$$

Here $P_{0}=1$. The first-step solution $P_{1}$ defines the leading approximation for the propagators of the gauge and ghost fields. The following steps define the many-particle functions.

\section{$3 \quad$ Solution of first-step equations}

The solution of first-step equations of the iteration scheme (9)-(10) is the quadratic polynomial in the sources

$$
P_{1}=\frac{1}{2 i} J * D * J+i \bar{\eta} * \Delta * \eta
$$


Eqs. (9) and (10) give us equations for the functions $D_{\mu \nu}^{a b}(x-y)$ and $\Delta^{a b}(x-y)$. The equation for $D_{\mu \nu}$ can be essentially simplified by modifying the gauge condition. Instead of the usual covariant gauge used above, it is convenient to use the modified gauge condition (" $V$ gauge") with the gauge fixing term

$$
\mathcal{L}_{\text {gauge }}=-\frac{1}{2 \alpha}\left(\mathcal{D}_{\mu}(V) A_{\mu}\right)^{2} .
$$

The ghost terms should be changed correspondingly. For the transition to the $V$-gauge in formulae (1)-(4), (5) and (9)-(10), it is sufficient to perform the substitution

$$
\partial_{\mu} \rightarrow \mathcal{D}_{\mu}(V)
$$

An essential circumstance is that the leading approximation condition (7) is not changed in the case.

The equations have a particularly simple form in the gauge $\alpha=1$ ("diagonal $V$-gauge"). Then, the equation for $D_{\mu \nu}$ in momentum space is

$$
\left\{\mathcal{K}^{2} g_{\mu \nu}+2\left[\mathcal{K}_{\mu}, \mathcal{K}_{\nu}\right]\right\} \tilde{D}_{\nu \lambda}(k)=-g_{\mu \lambda},
$$

where $k$ is the momentum, and the following notation has been introduced:

$$
\mathcal{K}_{\mu}^{a b}=k_{\mu} \delta^{a b}-W_{\mu}^{a b} .
$$

In the region of large $k$ eq. (14) tends to the equation for the free propagator in the diagonal gauge, i.e. at $k \rightarrow \infty$

$$
\tilde{D}_{\mu \nu}(k) \approx-\frac{1}{k^{2}} g_{\mu \nu} .
$$

The equation for the ghost propagator $\Delta$ is

$$
\mathcal{K}^{2} \tilde{\Delta}(k)=-1
$$

In the large $k$ region the propagator $\tilde{\Delta}$ also tends to the free propagator. Therefore, the ultraviolet behaviour of the solutions with a nontrivial vacuum vector $\mathbf{V}_{\mu}$ is the same as for the usual perturbation theory.

Below we consider the case of $S U(2)$ gauge group and restrict ourselves to the subset of constant $\mathbf{V}_{\mu}$ with zero field tensor $F_{\mu \nu}(V)=$ 0 , or, equivalently, with

$$
\epsilon^{a b c} V_{\mu}^{b} V_{\nu}^{c}=0
$$


It is clear that condition (18) ensures the leading approximation condition (7). The energy of that kind of solutions is zero, as for the perturbative solution with trivial vacuum. In that sense this set of solutions can be termed quasiperturbative. Eq. (18) gives us $\left[\mathcal{K}_{\mu}, \mathcal{K}_{\nu}\right]=$ $\left[W_{\mu}, W_{\nu}\right]=0$, and the solution of eq.(14) is reduced to the inversion of the matrix $\mathcal{K}^{2}$. The result is

$$
\tilde{D}_{\mu \nu}^{a b}(k)=-g_{\mu \nu}\left[\frac{p}{p_{1}} \delta^{a b}+\frac{2\left(k W^{a b}\right)}{p_{1}}+\left(\frac{1}{k^{2}}-\frac{p}{p_{1}}\right) \frac{\left(V^{a} V^{b}\right)}{V^{2}}\right],
$$

where the following notations has been introduced:

$$
p(k, V)=k^{2}+g^{2} V^{2}, p_{1}(k, V)=p^{2}-4 g^{2}(k V)^{2} .
$$

A solution of the ghost propagator equation (17) is also given by formula (19) (without $g_{\mu \nu}$ ).

Except for eqs. (14) and (17), the first-step equations give one more relation that contains a quantity $D_{\mu \nu}(0)$, which should be understood as some regularization. In essence this relation is a condition for the first step counterterms $\delta z_{1}$. (There is no need to introduce the leading approximation counterterms in this case, i.e. $\delta z_{0}=0$ ). Since the Green functions of the first step are finite, this condition for the counterterms is necessary to remove the ultraviolet divergences at the second step of the scheme. This peculiarity of the given iteration scheme is displayed here in exactly the same manner as for the scalar field theory (see [2]).

\section{Gauge field propagator}

Let us turn now to a possible physical interpretation of the solutions. At $\mathbf{V}_{\mu}=0 D_{\mu \nu}$ and $\Delta$ are free propagators of gauge and ghost field, and the whole iteration scheme is a reconstructed series of the perturbation theory. At $\mathbf{V}_{\mu} \neq 0$ the situation is more complicated. It is clear that in this case it is difficult to interpret the function $D_{\mu \nu}$ given by (19) as the propagator of a particle in the Poincaré invariant theory. Let us recall, however, that we have a number $\{G(V)\}$ of solutions of SDEs, each corresponding to some vector $\mathbf{V}_{\mu}$ satisfying the condition (18). Due to eq.(18) the field strength tensor is identically zero and any such solution possesses zero energy. Therefore, a candidate for 
the "physical vacuum functional" for this set of solutions is a superposition of all solutions. We shall exploit this fact in the construction of a Poincaré invariant solution which can be interpreted as a particle propagator, i.e. a function depending only on the momentum $k$ and the scalar quantity

$$
v^{2}=V^{2} \equiv V_{\mu}^{a} V_{\mu}^{a}
$$

(The quantity $v^{2}$ plays the role of an order parameter.) The construction is equivalent, in essence, to some averaging, i.e. an integration with a measure $d \mu(V)$, we shall therefore denote it by angular brackets: $\langle G\rangle=\sum_{V} G(V)$, etc. In the foundation of this operation we set the following conditions

$$
<V_{\mu}^{a}>=0, \quad<V^{2}>=v^{2} .
$$

Their necessity for the Poincaré invariant theory is evident. It is also evident that

$$
<V_{\mu}^{a} V_{\nu}^{b}>=\frac{1}{4} v^{2} g_{\mu \nu} E^{a b}
$$

where $\operatorname{tr} E=1$. To determine the form of the matrix $E^{a b}$, consider the leading approximation condition (18). The geometrical meaning of condition (18) is the collinearity of the vectors $\mathbf{V}_{\mu}$ in isotopic space. Consequently, at $\mathbf{V}_{\mu} \neq 0$ there exists a selected direction in isotopic space. This direction can be chosen as a basis vector, for example $\mathbf{n}_{3}$. In this basis $V_{\mu}^{a}=\delta^{a 3} v_{\mu}$, and $E^{a b}=\delta^{a 3} \delta^{b 3}$. Therefore, at $\mathbf{V}_{\mu} \neq 0$ the isotopic symmetry is spontaneously broken.

Further calculation is reduced to the averaging of the functions $f\left((k V)^{2}\right)=p / p_{1}$ and $f^{a b}=f \cdot\left(V^{a} V^{b}\right) / V^{2}$. First of all, note that at $k \rightarrow 0 f \rightarrow 1 / g^{2} v^{2}$, and at $k \rightarrow \infty f \rightarrow 1 / k^{2}$. These Poincaréinvariant properties of $f$ should, of course, be conserved for $\langle f\rangle$ as well.

With eqs.(22)-(24) and its generalizations for an arbitrary monomial in $\mathbf{V}_{\mu}$, the following formulae can be proved

$$
\begin{gathered}
<(k V)^{2 n}>=\left(k^{2} v^{2}\right)^{n} \frac{\Gamma(n+1 / 2)}{(n+1) ! \Gamma(1 / 2)}, \\
<(k V)^{2 n}\left(V^{a} V^{b}\right)>=E^{a b} v^{2}<(k V)^{2 n}>,
\end{gathered}
$$

which are necessary for the calculation of $\left\langle D_{\mu \nu}\right\rangle$. The result of the calculation in the above basis is as follows:

$$
<\tilde{D}_{\mu \nu}^{33}(k)>=-g_{\mu \nu} \frac{1}{k^{2}}
$$




$$
<\tilde{D}_{\mu \nu}^{11}(k)>=<\tilde{D}_{\mu \nu}^{22}(k)>=-g_{\mu \nu} \frac{k^{2}+g^{2} v^{2}}{2 g^{2} v^{2} k^{2}}\left(1-\sqrt{1-\frac{4 g^{2} v^{2} k^{2}}{\left(k^{2}+g^{2} v^{2}\right)^{2}}}\right)
$$

(Other isotopic components are equal to zero.) Therefore, along the selected isotopic direction the particle propagates as a free one, but along other directions a separation of the region of momenta exists, the scale of the separation being the quantity $g^{2} v^{2}$. It is necessary to stress that both the limiting cases above $\left(k^{2} \rightarrow 0\right.$ and $\left.k^{2} \rightarrow \infty\right)$ belong to the region of applicability of the calculations performed, which is defined by the condition $\left|4 g^{2} v^{2} k^{2} /\left(k^{2}+g^{2} v^{2}\right)^{2}\right|<1$. Consider the question about analytical continuation. Eq. (26) defines two analytical functions depending on the choice of branch of the function $\sqrt{z^{2}}$, but neither satisfies both the above asymptotical conditions simultaneously and, consequently, these functions are not solutions of the problem. Hence, as a solution one should choose at large $k$ the branch with the behaviour $1 / k^{2}$, and at small $k$ - another branch which is a constant $1 / g^{2} v^{2}$. At the points $\pm g^{2} v^{2}$ the solution goes from one branch to another, i.e. from the "perturbative" sheet to the "non-perturbative" one. At the point $k^{2}=g^{2} v^{2}$ the solution is continuous, and at the point $k^{2}=-g^{2} v^{2}$ has a discontinuity. (Note that the first-step calculations of the iteration scheme do not fix the sign of $v^{2}$.) Such unusual features of the solution near the separation points are likely to be connected with our limitation of the characteristic equation solutions. Probably an extension of the class of solutions will lead to smoothing of the propagator behaviour near the points of separation of the perturbative and non-perturbative regions.

\section{Discussion}

In this paper we have attempted to introduce a new iteration scheme to solve the Schwinger-Dyson equations for non-Abelian gauge theory. As a very first step we have considered a scheme based on the simplest constant solutions of the characteristic equations (5). Of course, there are no prior grounds for expecting such a set of solutions to be useful for the physical applications. Some other sets of more non-trivial solutions (instanton like and so on) of the characteristic equations are likely to be of real physical interest. Dealing with coordinate-dependent solutions is a more difficult task; in partic- 
ular, the averaging procedure of Sec. 4 should be essentially modified. Another important question is the correspondence of the proposed method with the functional integral approaches, such as well-known background field method [6]. In spite of their evident similarity, the proposed iteration scheme seems to be somewhat different: the structure of expansions is distinct. The problem of the correspondence requires further investigations based on the calculation of higher steps of the iteration scheme.

The set of quasiperturbative solutions of Sects. 3-4 leads to spontaneous breaking of $S U(2)$ gauge symmetry. The question arises as to whether such solutions can be used to construct a realistic model of electroweak interactions in the spirit of dynamical gauge symmetry breaking models without Higgs bosons (see [7] and refs. therein). Further investigation which should essentially extend the class of solutions is necessary to eliminate this question. On the other hand, the set of quasiperturbative solutions satisfying condition (18) considered here does not exhaust the solutions with zero energy, and some further averaging to restore the symmetry may be necessary.

For $S U(3)$ gauge group (the case of QCD) the variety of solutions increases greatly in comparison with $S U(2)$ group (see, for example, [8]) . Avoiding discussion of this case, nevertheless note that the infrared finite behaviour of the gluon field propagator has recently been discussed in detail (see [9] and refs. therein).

\section{Aknowlegements}

The author is grateful to A.I. Alekseev, B.A. Arbuzov, P.A. Saponov and V.V. Vladimirsky for useful discussions. The work is supported by RFBR, grant No.95-02-03704.

\section{References}

[1] Bender C M, Cooper F and Simmons L M, Jr. 1989 Phys.Rev. D 392343

Rochev V E 1993 J.Phys. A: Math.Gen. 261235

Garcia S, Guralnik Z and Guralnik G S 1996 preprint

Massachusetts Institute of Technology MIT-CTP-2582 (hepth/9612079) 
[2] Rochev V E 1997a J.Phys. A: Math.Gen. 303671

(hep-th/9606155)

[3] Rochev V E and Saponov P A 1997 hep-th/9710006 (to be published in Int.J.Mod.Phys. A)

[4] Rochev V E 1997b in "Quantum Chromodynamics: Collisions, Confinement, Chaos" eds H M Fried and B Muller (Singapore: World Scientific) p.354 (preprint IHEP Protvino 96-61)

[5] 't Hooft G 1982 Phys.Scripta 25133

[6] Abbott L F 1981 Nucl.Phys. B 185189

[7] Arbuzov B A 1995 Phys.Lett. B 353513

[8] Vladimirsky V V 1996 Yad.Fiz. 592063

[9] Cudell J R and Ross D A 1991 Nucl.Phys. B 359247

Kiselev V V 1994 Mod.Phys.Lett. A 92421

Alekseev A I 1996 Teor.Mat.Fiz. 106250 